\title{
An RF Predistorter for Base Station HPAs of NADC System
}

\author{
M. X. Xiao, S. W. Cheung and T. I. Yuk \\ The Department of Electrical and Electronic Engineering \\ The University of Hong Kong \\ Hong Kong \\ mxxiao@eee.hku.hk,swcheung@eee.hku.hk,tiyuk@eee.hku.hk
}

\begin{abstract}
This paper presents the design of an effective and low cost RF predistorter for use in the base-station high power amplifiers (HPAs) of the North American Digital Cellular (NADC) system. A complex polynomial is used to model the characteristic of the HPA and the predistorter generates the inband intermodulation (IM) products required for predistortion of the HPA. The design of the predistorter has a simple structure, using Schottky diodes as the nonlinear device to generate the inband IM products. Experimental results using a practical HPA show that the predistorter can reduce the adjacent-channelpower ratio (ACPR) of the pi/4-DQPSK signal used in the NADC system by $20 \mathrm{~dB}$ at an HPA output power level of $30 \mathrm{~W}$. Moreover, the constellation and eye diagrams of the pi/4-DQPSK signal show that the predistorter can also reduce the signal distortion caused by the HPA.
\end{abstract}

Index terms - Adjacent-channel-power ratio (ACPR), Constellation, Eye diagram, pi/4-DQPSK, High Power Amplifier, Mixer, RF Analog Predistorter.

\section{INTRODUCTION}

The major source of intermodulation distortion in the RF front end of a base station transmitter in a mobile system is the high power amplifier (HPA). There is an increasingly stringent linearity requirement for HPAs in wireless communication systems. The first generation of digital cellular mobile system, IS-54, employs a spectrally efficient modulation scheme, pi/4-DQPSK. However, achieving spectral efficiency requires the use of a linear transmit power amplifier [1]. In modern communication standards such as CDMA-2000 and WCDMA, the RF signals have high peakto-average ratios, requiring the HPAs to be stringently linear. Due to the inherent nature of RF HPAs, the amplification processes are always nonlinear. In order to keep the intermodulation distortion at the acceptable levels, the HPAs are needed to operate at large back-off from saturation [2], or some linearization techniques must be adopted.

Among various linearization techniques, digital predistortion (DPD) technique has been actively studied in the recent years because of high linearity improvement, but it is complicated and typically implemented in the base-band level [3]. While analog predistortion technique is more suitable for repeater systems because of the ability to directly amplify the
RF signals between handsets and base-stations [4]. Moreover, analog predistortion has advantages of simpler structure, lower cost and having a much wider bandwidth.

The principle of RF predistortion is to have a characteristic complementary to that of the RF HPA. An RF predistorter can be implemented by generating either the harmonic-frequency signals [5] or the inband intermodulating (IM) products [6-7] and then adding to the original signal at the input of the HPA. In general, there are two ways, i.e. using the differencefrequency signal or the sum-frequency signal, to generate the inband IM products [6, 7]. For example, a diode can be used as a nonlinear device to generate the difference-frequency signal [6]. However, for HPAs operating in the microwave frequency band, the difference-frequency signal will be out of the HPA operation frequency band and thus blocked. Two wideband double-balanced mixers capable of operating at twice of the maximum frequency can also be used to generate the sum-frequency signal in order to generate the IM3 signals [7]. But it is not easy to handle signals with such high frequency, particularly when generating the IM products with higher orders for intermodulation distortion (IMD) products cancellation.

This paper describes the design of an effective and low cost predistorter employing two mixers as the nonlinear devices to generate the inband IM products for predistortion of base station HPAs in the North American Digital Cellular (NADC) system. The hardware implementation of the design is tested using a pi/4-DQPSK signal on a practical HPA. In [8], a predistorter using inband intermodulation frequencies has been proposed with modulated CDMA IS-95 signals spectrum measurements, but the effects of the HPA linearity improvement on the system were not considered. Here, we demonstrate that, at an HPA power level of $30 \mathrm{~W}$, the predistorter can suppress the adjacent-channel-power ratio (ACPR) of the signal spectrum by $20 \mathrm{~dB}$. Moreover, the constellation and eye diagrams from the demodulated signals are also studied in this paper. Results show that the predistorter can reduce the signal distortion, hence improve the bit-error rate of the signal in the NADC system.

The remainder of this paper is organized as follows. Section II describes the model of HPA and predistortion. The circuit 
design of the RF predistorter is described in section III. Simulation and measurement results with discussions are given in section IV. Finally, conclusions are drawn in section $\mathrm{V}$.

\section{MODEL OF HPA AND PREDISTORTION}

The nonlinear process of an HPA can be written as:

$$
y=A(x) e^{j \phi(x)}=A(x) \cos (\phi(x))+j A(x) \sin (\phi(x))
$$

where $\mathrm{x}$ is the input signal, $\mathrm{y}$ is the output signals, $A($.$) and$ $\phi($.$) are the amplitude-amplitude (AM-AM) and amplitude-$ phase (AM-PM) distortion effects, respectively, of the HPA. Using a complex polynomial, the nonlinear process of (1) can be approximated by:

$$
\begin{aligned}
y & =\left(a_{1} x+a_{2} x^{2}+a_{3} x^{3}+\ldots\right)+j\left(b_{1} x+b_{2} x^{2}+b_{3} x^{3}+\ldots\right) \\
& =\sum_{i=1}^{\infty}\left(a_{i}+j b_{i}\right) x^{i}
\end{aligned}
$$

where $a_{1}, a_{2}, a_{3}, \ldots$ and $b_{1}, b_{2}, b_{3}, \ldots$ are the coefficients of the real and imaginary polynomials, respectively.

The $3^{\text {rd }}$-order nonlinearity is the dominant HPA nonlinearity, thus the approximation of (1) with (2) can be performed by using a $3^{\text {rd }}$-order polynomial representation,

$$
y=\left(a_{1} x+a_{2} x^{2}+a_{3} x^{3}\right)+j\left(b_{1} x+b_{2} x^{2}+b_{3} x^{3}\right)
$$

The even-order terms produce IM products which are outside the HPA operation frequency band and do not contribute to inband distortion, so we can consider only the odd-order terms in (3). Assume the input is a two-tone signal with equal amplitude and zero initial phase, and given by $x=\cos \left(\omega_{1} t\right)+\cos \left(\omega_{2} t\right)$. Substituting $x$ into (3) yields a large number of terms. The even-order terms produce the intermodulation distortion products (IMDPs) which in frequency are away from the original two-tone signal and do not contribute to inband signal distortion, so we only need to consider the odd-order terms which produce the IMDPs coincident with the original two-tone signal. It can easily be shown that the $3^{\text {rd }}$-order terms in the real and imaginary polynomials of (3) generate the $3^{\text {rd }}$-order intermodulation distortion (IMDP3) products:

$$
\begin{aligned}
& \operatorname{IMDP}(t)=3 a_{3}\left\{\cos \left[\left(2 \omega_{1}-\omega_{2}\right) t\right]+\cos \left[\left(2 \omega_{2}-\omega_{1}\right) t\right]\right\} / 4+ \\
& j 3 b_{3}\left\{\cos \left[\left(2 \omega_{2}-\omega_{1}\right) t\right]+\cos \left[\left(2 \omega_{1}-\omega_{2}\right) t\right]\right\} / 4
\end{aligned}
$$

and other IMDPs with higher orders.

To cancel the IMDPs, we generate the $3^{\text {rd }}$-order intermodulation (IM3) signal,

$$
\begin{aligned}
& \operatorname{IM}(3)=\left[\cos \left(2 \omega_{2}-\omega_{1}\right) t+\cos \left(2 \omega_{1}-\omega_{2}\right) t\right]+ \\
& j\left[\cos \left(2 \omega_{2}-\omega_{1}\right) t+\cos \left(2 \omega_{1}-\omega_{2}\right) t\right]
\end{aligned}
$$

adjust the amplitudes and phases, and then add it to the original input signal to produce:

$$
\begin{aligned}
& \cos \left(\omega_{1} t\right)+\cos \left(\omega_{2} t\right) \\
& +k_{1}\left[\cos \left(\left(2 \omega_{2}-\omega_{1}\right) t+\phi_{1}\right)+\cos \left(\left(2 \omega_{1}-\omega_{2}\right) t+\phi_{1}\right)\right] \\
& +j k_{1}^{\prime}\left[\cos \left(\left(2 \omega_{2}-\omega_{1}\right) t+\phi_{1}^{\prime}\right)+\cos \left(\left(2 \omega_{1}-\omega_{2}\right) t+\phi_{1}^{\prime}\right)\right]
\end{aligned}
$$

where $k_{1}, k_{1}^{\prime}$ and $\phi_{1}, \phi_{1}^{\prime}$ are the adjusted amplitudes and phases, respectively. The resultant signal is fed to the HPA. Substituting (6) into (3) gives the $3^{\text {rd }}$-order IMD at the HPA output:

$$
\operatorname{IMDP} 3(t)=\left(f_{3}\left(k_{1}, k_{1}^{\prime}, \varphi_{1}, \varphi_{1}^{\prime}\right)+j g_{3}\left(k_{1}, k_{1}^{\prime}, \varphi_{1}, \varphi_{1}^{\prime}\right)\right) \cos \left(\left(2 \omega_{m}-\omega_{n}\right) t\right)
$$

having $m=1, n=2$ and $m=2, n=1$. Thus the IMDPs can be reduced by adjusting $\phi_{1}, \phi_{1}^{\prime}, k_{1}$ and $k_{1}^{\prime}$ with phase shifters and attenuators.

\section{CirCUit Design OF PREDISTORTER}

The circuit diagram of our proposed IM3 generator is shown in Fig. 1, which consists of two mixers. where RF\#1, LO\#1, $\mathrm{RF} \# 2$ and $\mathrm{LO} \# 2$ are input ports, IF\#1 and IF\#2 are output ports, and $\mathrm{RF}_{1}, \mathrm{LO}_{1}, \mathrm{IF}_{1}, \mathrm{RF}_{2}, \mathrm{LO}_{2}$ and $\mathrm{IF}_{2}$ are the signals in these ports.

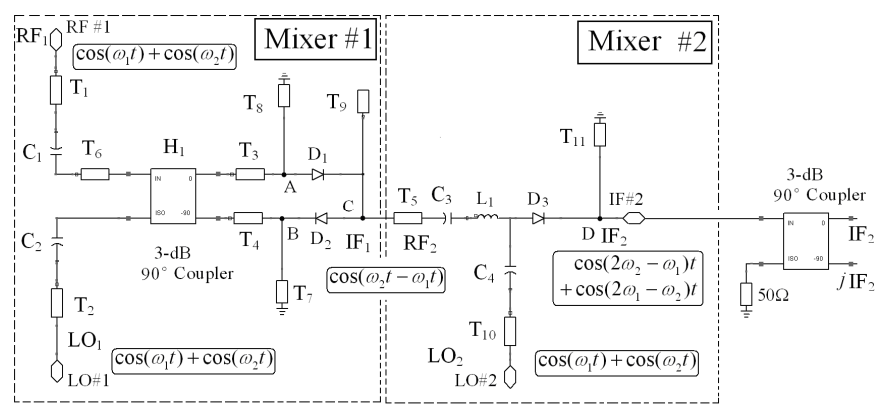

Fig. 1 Circuit diagram for IM3 generation

Mixer \#1 is a single-balanced mixer. $\mathrm{C}_{1}$ and $\mathrm{C}_{2}$ are the filter capacitors used to block the dc component, and $T_{1}$ and $T_{2}$ are $50-O h m$ transmission lines with equal length serving as the input ports, RF\#1 and LO\#1, to which the two signal $\cos \left(\omega_{1} t\right)+\cos \left(\omega_{2} t\right)$ are fed. $\mathrm{T}_{6}$ is a quarter-wave transmission line at the frequency $\omega_{1}$ (assume $\omega_{1} \approx \omega_{2}$ ), so the signal at the input of the $3-\mathrm{dB} 90^{\circ}$ hybrid coupler $\left(\mathrm{H}_{1}\right)$ has a $90^{\circ}$ phase delay. At the balanced ports of the coupler, the signals in RF\#1 and LO\#1 have the same phase in $T_{4}$, but a phase difference of $180^{\circ}$ in $T_{3}$. An antipodal diode pair, using Schottky diodes $\mathrm{D}_{1}$ and $\mathrm{D}_{2}$, is connected to the outputs of the coupler. The mixing IF components in each diode element with equal phase are combined together to form the output signal $\cos \left(\omega_{2}-\omega_{1}\right) t$ in IF\#1 and those with a phase difference of $180^{\circ}$ are cancelled off. $\mathrm{T}_{7}$ and $\mathrm{T}_{8}$ are quarter-wave shortcircuited lines at $\omega_{1}$. Since $\omega_{1} \approx \omega_{2}$, points $A$ and $B$ are seen 
as an open circuit by the signals in RF\#1 and LO\#1 which are fed to the diodes for mixing. The dc signal produced by the mixing process is used to bias the diodes $\mathrm{D}_{1}$ and $\mathrm{D}_{2}$, but blocked by the capacitor $\mathrm{C}_{3}$. Other high order harmonic frequency mixing components are short circuited by $T_{9}$. The signal $\cos \left(\omega_{2}-\omega_{1}\right) t$ in IF\#1, being at a much low frequency, sees points $\mathrm{A}$ and $\mathrm{B}$ as a short circuit. $\mathrm{T}_{9}$ is a quarter-wave open-circuited transmission line also at $\omega_{1}$, so point $\mathrm{C}$ is also seen as a short circuit for signals $\cos \left(\omega_{1} t\right)+\cos \left(\omega_{2} t\right)$ in RF\#1 and $L O \# 1 . T_{3} \& T_{8}$ and $T_{4} \& T_{7}$ also form the matching networks for diodes $\mathrm{D}_{1}$ and $\mathrm{D}_{2}$, respectively, to reduce the mixer's conversion loss [9].

Mixer \#2 is a single-ended mixer. The output signal $\cos \left(\omega_{2}-\omega_{1}\right) t$ from IF\#1 is fed via $\mathrm{T}_{5}$ to $\mathrm{RF} \# 2$ of Mixer \#2. $\mathrm{C}_{3}$ and $\mathrm{L}_{1}$ form a tune circuit to block the de signal and pass the signal to diode $\mathrm{D}_{3}$ for mixing The tune circuit also blocks the high-frequency signals from LO\#2 and IF\#2. $\mathrm{T}_{10}$ and $\mathrm{C}_{4}$ form a high-pass filter to pass the wanted IM3 signal $\cos \left(2 \omega_{1}-\omega_{2}\right) t+\cos \left(2 \omega_{2}-\omega_{1}\right) t \mathrm{LO}_{2}$ and block the lowfrequency signal. $\mathrm{T}_{11}$ is a $50-\mathrm{Ohm}$ quarter-wave shortcircuited transmission line at $\left(2 \omega_{2}-\omega_{1}\right)\left(\right.$ or $\left.\left(2 \omega_{1}-\omega_{2}\right)\right)$ and so point $\mathrm{D}$ is seen by the signal $\cos \left(2 \omega_{1}-\omega_{2}\right) t+\cos \left(2 \omega_{2}-\omega_{1}\right) t$ in IF\#2 as an open circuit and the low-frequency signal as a short circuit. This mixer circuit does not need any dc supply, so it is simple, low cost and easy to implement. The wanted IM3 signals from Mixer \#2 are fed to a $90^{\circ}$-coupler, as shown in Fig. 2. The output from Combiner $\# 1$ is combined with the original two tone signal in Combiner \#2 to product the resultant signal to the HPA.

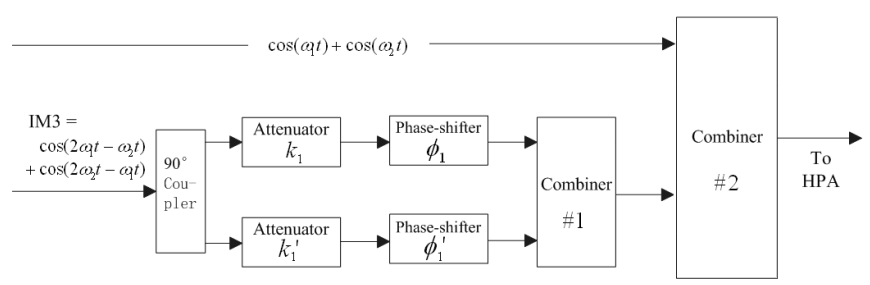

Fig. 2 Generation of predistorted signal

\section{RESULTS}

Simulation tests using Agilent ADS 2008A have been used to assess the performance of the predistorter on a practical base station HPA, HW-1900-40, manufactured by Bravotech inc, China. The characteristic of the HPA was first modeled using a complex polynomial and then used in ADS 2008A. A two-tone signal at the frequencies of 2.2 and $2.21 \mathrm{GHz}$ was used to obtain the correct settings for the phase shifters and attenuators as follows. The two-tone signal was applied to the inputs of the predistorter. The phase shifters and attenuators were adjusted to minimize the powers of the $3^{\text {rd }}$-IMD products at the HPA output. With the settings of the phase shifters and attenuators unchanged, a pi/4-DQPSK signal used in the NADC system was used to apply to the input of the predistorter. The simulation results on the signal spectra from the HPA output with and without the predistorter are shown in Fig. 3. It can be seen that the predistorter can significantly reduced the ACPR suppression by $20 \mathrm{~dB}$ at the band edge of $20 \mathrm{kHz}$.

The predistorter has also been fabricated on a PCB using Roger's RO4005C. The 3-dB 90 ${ }^{\circ}$ hybrid coupler used was Anaren's model JP503S and the Schottky diodes were Avagotech's HSMS 282X. The chip capacitors $\mathrm{C}_{1}, \mathrm{C}_{2}, \mathrm{C}_{3}$ and $\mathrm{C}_{4}$ had values of $100 \mathrm{pF}, 100 \mathrm{pF}, 10 \mathrm{nF}$ and $100 \mathrm{pF}$, respectively, with a mounting size of 0603 (60 mil x $30 \mathrm{mil})$. The inductor $\mathrm{L}_{1}$ had a value of $100 \mathrm{nH}$ with a mounting size of 0805 (80 mil x 50 mil).

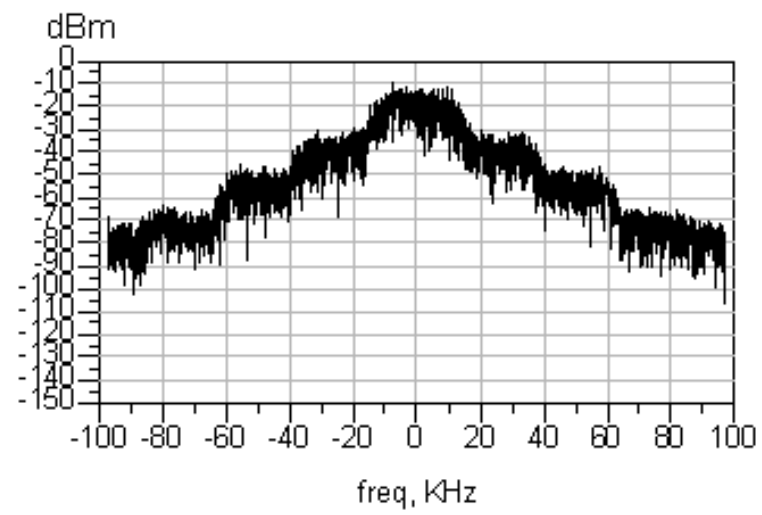

(a)

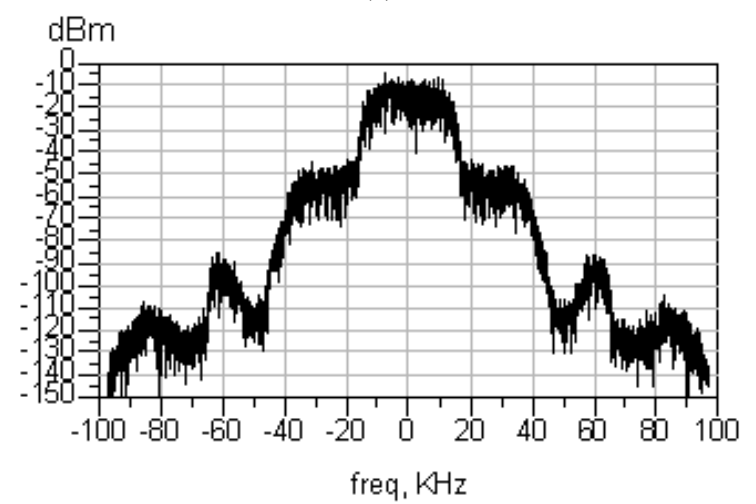

(b)

Fig. 3 Simulated NADC signal spectra from HPA:

(a) without predistortion and (b) with predistortion

To assess the predistortion performance of our hardware design, the same practical base station HPA, HW-1900-40 manufactured by Bravotech inc. China, was used with a pi/4DQPSK generated using an R\&S SMIQ Vector Signal Generator as the input. Before measurement was taken, a twotone signal was again used to obtain the correct settings for the phase shifters and attenuators. In the two-tone test, two tone signals of 2.2 and $2.21 \mathrm{GHz}$ were generated using two signal generators and applied to the inputs of the predistorter. The phase shifters and attenuators were adjusted manually to minimize the powers of the $3^{\text {rd }}$-IMD products. The pi/4DQPSK signal was then fed to the inputs of the predistorter. 
The output powers from the HPA in these two tests were kept the same at $30 \mathrm{~W}(14.8 \mathrm{dBW})$. The measurement results of the spectra obtained via a $40-\mathrm{dB}$ coupler in the HPA, with and without the predistorter, are shown in Fig. 4. The predistorter achieves a $20 \mathrm{~dB}$ ACPR suppression at the band edge of 20 $\mathrm{kHz}$. The signal distortions caused by the HPA with and with the predistorter were also studied by demodulating the pi/4DQPSK signal using a vector signal analyzer. The eye diagram and signal constellation of the demodulated signal from the vector signal analyzer are shown in Figs. 5 and 6, respectively. It can be seen from these results that, with the use of the predistorter, the eye diagram is open wider and the signal constellation is lying on a larger circle, implying that, in addition to ACPR suppression, the predistorter can also reduce signal distortion and so improve the error-rate performance of the signal in the NADC system.

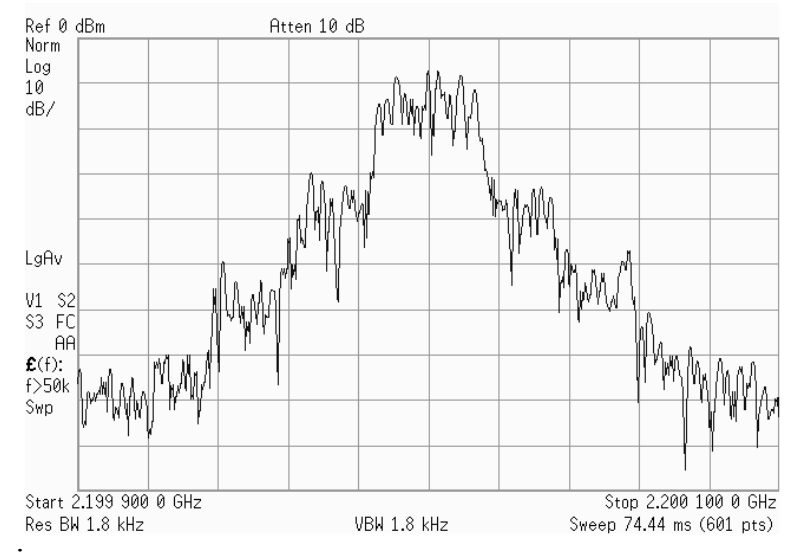

(a)

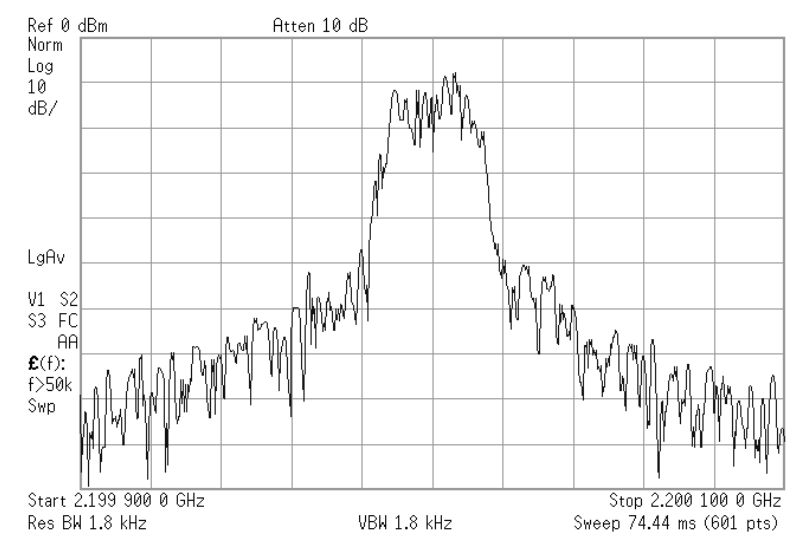

(b)

Fig. 4 Measured NADC signal spectra from HPA:

(a) without predistortion and (b) with predistortion

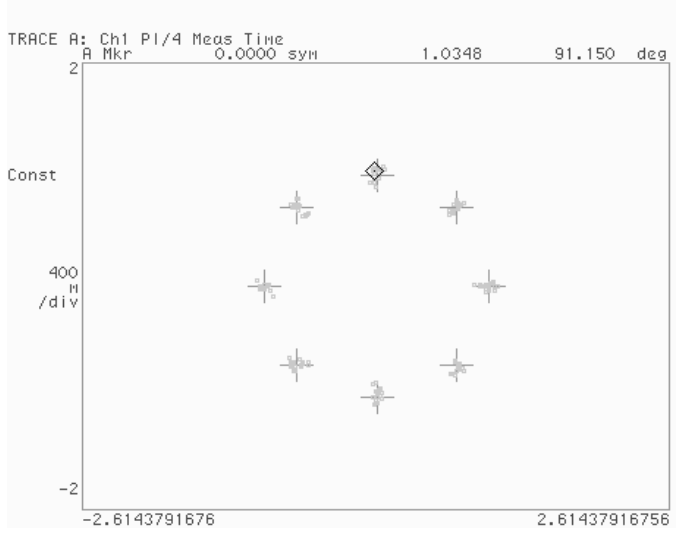

(a)

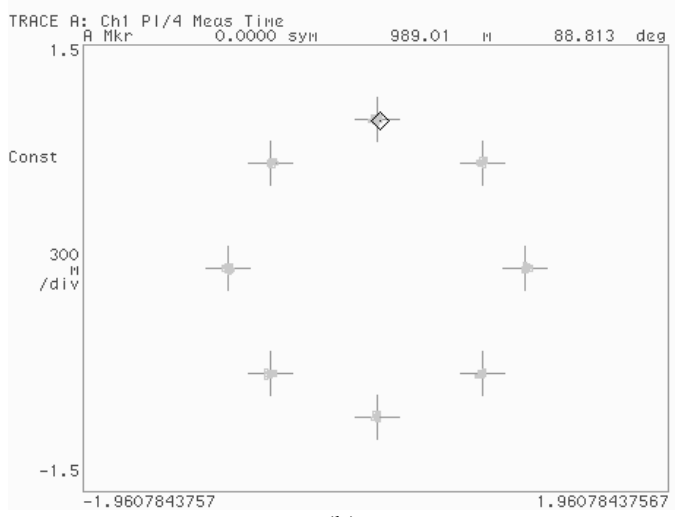

(b)

Fig. 5 Measured signal constellation of NADC signal from HPA: (a) without predistortion (b) with predistortion 


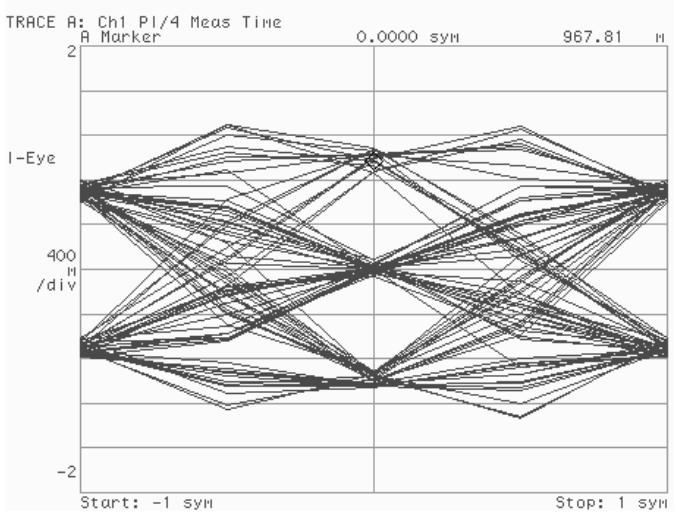

(a)

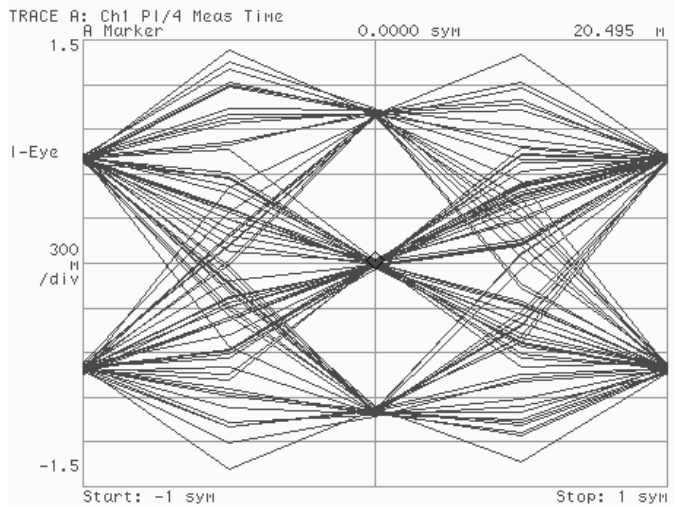

(b)

Fig. 6 Measured eye-diagram of NADC signal from HPA: (a) without predistortion and (b) with predistortion

\section{CONCLUSIONS}

The design of an effective and low cost and RF predistorter has been presented in the paper. Experimental results have shown that the predistorter has worked very effectively on a practical HPA operating at $2.2 \mathrm{GHz}$. For the pi/4-DQPSK signal used in the NADC system, the predistorter can suppress the ACPR by $20 \mathrm{~dB}$ at the HPA output at a power level of 30 W (14.8 dBW). Results on signal constellation and eye diagram have also shown that it can reduce the signal distortion caused by the HPA and hence improve the bit-error rate of the pi/4-DQPSK signal

\section{REFERENCES}

[1] J. Boccuzzi, "Performance Evaluation of Non-linear Transmit Power Amplifiers for North American Digital Cellular Portables", IEEE Transactions on Vehicular Technology, Vol. 44. No. 2, pp. 220-228, May 1995.

[2] A. A. Kidwai, and B. Mali, "Power Amplifier Predistortion Linearization using a CMOS polynomial generator", IEEE Radio Frequency Integrated Circuits (RFIC) Symposium, pp. $255-258,2007$
[3] S.Y. Lee, Y.S. Lee, and Y. H. Jeong etc, "Fully-automated adaptive analog predistortion power amplifier in WCDMA applications", European Microwave Conference pp.1-4, 2005

[4] S. Y. Lee, Y. S. Lee, and S. H. Hong etc, "An Adaptive Predistortion RF Power Amplifier with a Spectrum Monitor for Multicarrier WCDMA Applications," IEEE Trans. Microwave Theory and Techn., vol. 53, no. 2, pp. 786-793, Feb. 2005

[5] K. J. Cho, et al, "Multi-order predistortion of power amplifiers using a second harmonic based technique," IEEE Microwave and Wireless Components Letters, Vol. 13, pp. 452-454, Oct. 2003.

[6] M. Modeste, D. Budimir, M. Moazzam, and C. S. Aitchison, "Analysis and practical performance of a difference frequency technique for improving the multicarrier IMD performance of RF amplifiers," IEEE MTT-S Symp. Dig., pp. 53-56, Feb. 1999.

[7] T. Nesimoglu, C. N. Canagarajah, and J. P. McGeehan, "A broad band polynomial predistorter for reconfigurable radio", VTC 01, pp. 1968-1972, 2001.

[8] M. X. Xiao, S. W. Cheung, and T. I. Yuk, "A simple and effective RF predistorter for use in the HPAs of base stations in cellular mobile systems", ICCS08, pp. 805-808, 2008.

[9] Y. H. Liew, and J. Joe, "RF and IF ports matching circuit synthesis for a simultaneous conjugate-matching mixer using quasi-linear analysis," IEEE Transactions on Microwave Theory and Technique, Vol. 50, No.9, pp. 2056-2062, 2002. 